\title{
Application of Wavelet Neural Network to Fault Diagnosis of Power System
}

\author{
Hu Jian, Hu Fanjun
}

The First Aeronautic Institute of the Air Force, Xinyang, 464000, China

Keywords: application; neural network; fault diagnosis; power system.

\begin{abstract}
Based on analyzing the incompleteness and uncertainty of information existing in power system fault diagnosis, a new fault diagnosis method based on wavelet transform and neural network is proposed. The wavelet transform is used to pre-process data and extract feature vectors. The neural network is used to identify fault types. Diagnostic results of instance proved the effectiveness and superiority of the proposed method.
\end{abstract}

\section{Introduction}

With the rapid development of scientific technology, the scale and structure of power system continue to expand and become complicated. In the process of power system operation, natural and man-made interference often occurs and the failure is difficult to avoid. Therefore, adopting effective method to diagnose the fault of power system accurately, finding out the fault components, reducing manpower, material resources and economic losses, appear particularly important. Now, with the further development of artificial intelligence, especially machine learning, data mining, etc, many theories and methods are offered to diagnose the fault. Such as expert system, optimization method, fuzzy sets theory etc. Although these theoretical researches have scored some achievements, there are still certain limitations. For example, Fourier transform has been playing an important role in data processing, but Fourier transform has some defects, on the one hand, it can only analyze stationary signals, it cannot characterize sharp-variation signals that occur during faults diagnosing. On the other hand, Fourier transform cannot localize the singularities that always symbolize some sudden faults, and its frequency and time resolutions contradict each other.

As a powerful tool of signal analysis, wavelet transform has good localization properties in time and frequency domain, focus to any details of the analysis object with taking fine time or frequency step length of high frequency, express any changes existing in the object, so as to get accurate feature separation results from the measurement data with bad SNR[1-5].

By using wavelet transform to separate the feature, the key process lies in the determination of optimal decomposition levels. On the one hand, we want to separate the feature components as far as possible, on the other hand, keep the fixed errors and true value apart from the separated feature. The current methods need either manual setting threshold control or results testing with extracted trend by wavelet transform, which increase the difficulty of the application of separation methods and raise the risk of error introduced. In accordance with the above case, the paper proposes a new method which approximates the feature with detail components of the wavelet decomposition, determines the optimal decomposition level on the frequency intervals between the feature and other components, then gets the feature directly. The method avoids the indirect error with modeling and indirect methods [6, 7].

The uncertainty of power system operation, the diversity, complexity and associated leveloriented of gathering information, cause detection randomness and uncertainty. Wavelet is a new developing signal processing means. It is localized both in time and frequency domains. So it is possible to characterize the local singularities based on the coefficients in a wavelet orthonormal basis expansion. Combining model theory and statistical knowledge, wavelet provides a method to describe causal relationship between variables. Using probability theory to handle the uncertainty between different knowledge for conditions related, so it thus becomes one of the models in the field of uncertain knowledge representation and reasoning. Applying the neural network to power system fault diagnosis, can solve incomplete and uncertainty. Using the neural network structure 
learning algorithm to obtain a precise power system fault diagnosis model in qualitative, using the wavelet parameters learning algorithm to obtain the table of conditional probability and reflect the link degree between components in quantitative. Through reasoning algorithm further achieve the power system fault diagnosis under the uncertainty and incomplete information $[8,9]$.

\section{Wavelet transform}

Wavelet transform is made up of wavelet decomposition and wavelet reconstruction [10]. The decomposition process can be iterated with successive approximations being decomposed in turn, so that one signal is broken down into many lower resolution components. This is called the wavelet decomposition tree.

The tree diagram of signal $\mathrm{S}$ is after three levels wavelet decomposition is shown in Fig.1. S denotes the signal to be decomposed, Ai denotes approximations components of decomposition, and Di denotes detail components of decomposition. The subscript expresses the decomposition levels.

Reconstruction is the inverse process of wavelet decomposition. First of all, reconstruction the approximation and detail components on the lowest level to get approximation components of the last level. Repeat this process till recovering the original signals.

Between decomposition and reconstruction we may modify the wavelet coefficients before performing the reconstruction step. We perform wavelet analysis because the coefficients thus obtained have many known uses compression being foremost among them.

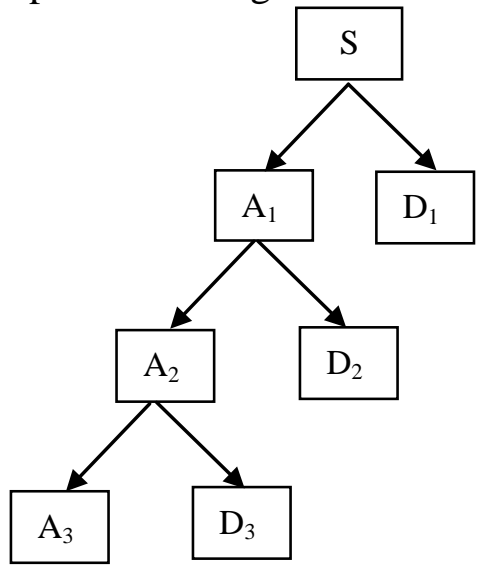

Fig.1: Tree diagram of three levels wavelet decomposition

Through the definition of wavelet basic function, the graph center of the function is similar with a periodic function and attenuates quickly outside that, which can be considered as a window function with a certain frequency character as in [9]. If a signal's frequency is close to the wavelet basic function, the scale of the signal in the window will be amplified, attenuated on the contrary. With the shifts and expansions of wavelet basic function, we can recognize the signal's frequency characters. Unlike the windowed Fourier transform, high frequency wavelets are very narrow, while low frequency wavelets are much broader. As a result, the wavelet transform is better able than the windowed Fourier transform to "zoom in" on very short-lived high frequency phenomena, such as transients in signals.

The existing methods of random errors separation which using wavelet transforms mainly extract trends of the measurement data and get the random errors by subtracting the trend items, which is difficult to apply because it requires the precise modeling of the true values and fixed error. The paper implements the separation from another perspective, which directly uses the details of wavelet transform approximate the random error of measurement data, determines the wavelet decomposition levels based on the frequency bands character of the measurement data and separates the random errors directly which avoiding additional errors from the modeling process. The new method of this paper need not know the error models that the random errors or the fixed errors satisfy with. The only thing we should pay attention to is setting up the relationship between the frequency bands character of measurement data and the decomposition levels of the wavelet 
transform, and finding out the optimal decomposition level when the random errors has been separated entirely.

If a function $\psi(\mathrm{t})$ satisfies the following admissibility condition, it is called mother wavelet.

$$
\int_{0}^{+\infty} \frac{\psi^{2}(\omega)}{\omega} d \omega=\int_{-\infty}^{0} \frac{\psi^{2}(\omega)}{\omega} d \omega<+\infty
$$

families of functions

$$
\psi_{a, b}=|a|^{-\frac{1}{2}} \psi\left(\frac{t-b}{a}\right)
$$

generated from $\psi(t)$ by the operation of dilation and translations form a basis or a frame of a square integrable space $L^{2}(R)$. Such families are called wavelets. Wavelet transform is to expand a signal to this wavelet.

$$
W(a, b)=\int_{-\infty}^{+\infty} \psi_{a, b}(t) f(t) d t
$$

$W(a, b)$ is the wavelet transform of $f(t)$. Here $a$ is called scale parameter and $b$ translation parameter. In order to calculate wavelet transform fast on computer, we must discretizate parameters $a$ and $b$. In digital signal processing, the dyadic discrete wavelet transform is most widely used. Make $a=2^{-j}, b=2^{-j} k$, where $j, k \in Z$. From the theory of the Multiresolution Analysis developed by Mallat, supposed that $\left\{V_{j}\right\}(j \in Z)$ is a multiresolution analysis of $L^{2}(R)$. There must be an unique scale function $\varphi(t)$, by dilation and translation of $\varphi(t)$, we can get a family of functions forming an orthonormal basis of $V_{j}$. The mother wavelet $\psi(t)$ can be deduced from $\varphi(t)$. And the family of functions forms an orthonormal basis of another set of closed sub-spaces $W_{j}$. $W_{j}$ is the orthogonal complement of $V_{j}$ in $V_{j+1}$, that is,

$$
W_{j} \perp V_{j}, W_{j} \oplus V_{j}=V_{j+1}
$$

Mallat proved that the resolution of any actual signals can be normalized to 1 , that is, $f(t) \in V_{0}$. So $f(t)$ can be projected to the set of the sub-space to yield its orthogonal wavelet representation $A_{j}^{d} f(n), D_{j}^{d} f(n)$, where $A_{j}^{d} f(n)$ is the projection of $f(t)$ on $V_{j}$, called approximation of $f(t)$. It can be interpreted as a low-pass filtering of $f(t), D_{j}^{d} f(n)$ is the projection of $f(t)$ on $W_{j}$, called detail signal. It can be interpreted as the filtering of $f(t)$ by a bank of band-pass filters. So wavelet transform can be viewed as extracting information on different frequency bands from the original signal and shows it on time axis. Thus we can view the signal characteristics on both time and frequency domains simultaneously. The high frequency information is shown on fine scales and the low frequency information on large scales. We can select transforms on proper scales to characterize the original signal according to our requirement. On this account wavelet transform is a powerful signal processing tool.

On the basis of the Multiresolution, Mallat developed a pyramid algorithm for discrete dyadic wavelet transform.

$$
\begin{aligned}
& A_{j}^{d} f(n)=\sum_{k=-\infty}^{\infty} h(2 n-k) A_{j}^{d} f(k) \\
& D_{j}^{d} f(n)=\sum_{k=-\infty}^{\infty} g(2 n-k) A_{j}^{d} f(k)
\end{aligned}
$$

It has been proved that we can reconstruct the original signal completely from its wavelet representation $A_{j}^{d} f(n), D_{j}^{d} f(n)$. This indicates that wavelet transform contains almost all of the original signal information. So it is viable to extract features by wavelet transform. From DWT if there are $\mathrm{N}$ data in the original signal, the lengths of $A_{j}^{d} f(n)$ and $D_{j}^{d} f(n)$ are $2 \mathrm{jN}(\mathrm{j}<0)$ respectively. That is, with increase of the scale, their lengths decrease in proportion to 2. Therefore, wavelet transform can compress data efficiently.

In fault diagnosis, the length of the feature vectors should be as small as possible under the condition of characterizing the faults accurately. Considering singularities of the fault signals, we take advantage of wavelet transform that is localized on both time and frequency domains, to extract a certain period as our diagnosing feature vector. To compare with each other, we study the following two feature extracting measures, the first is to extract $A_{-1}^{d} f$ as a feature vector. The difference between original signal and $A_{-1}^{d} f$ is $D_{-1}^{d} f . D_{-1}^{d} f$ mostly contains the noise information in 
high frequency domain. When it is discarded, SNR will increase greatly, and the data number will decrease half. The second is to extract $A_{-2}^{d} f$ from $A_{-1}^{d} f$. Together with the mean value, mean square value and variance of $A_{-2}^{d} f$ which characterize the lower frequency information of $A_{-1}^{d} f, A_{-2}^{d} f$ constructs another type of feature vector. Using this vector we will lose some information in low frequency domain, but its dimension is only about a quarter of that of the original signal[11].

When we regard these two feature vectors as the learning and testing sets of neural network system, the number of the neurons will decrease greatly comparing with choosing the original signal as the input samples. It has been turned out by some experiments that these measures are efficient and accurate in power system fault diagnosis.

\section{Neural network}

The application of the neural network to the fault diagnosis of power systems, bases on the preprocessing method by means of the learning function, the large-scale parallel distributed memorizing and processing capabilities, as well as the collective operating capability of the neural network, accomplishes the fault diagnosis of power systems. At last, accomplishes the adaptive inference and improve the real-time processing ability and adaptability of the power systems.

The schematic diagram of the application of neural network to the power system fault diagnosis is shown in Fig.2.

In the neural network fault diagnosis of power system, at first, we should build the neural network model. Secondly, decide the learning algorithm according to the characteristic of the power system. Finally, study the problems relating to the fault diagnosis. At present, there are many neural network models, we can select the self-organizing neural network and the multi-level feed forward neural network model. The ART (Adaptive Resonance Theory) neural network model can be adopted to perform the multiresolution. Now, we adopt the ART neural network model in diagnosing the fault of power system[12].

ART neural network model consists of the input and output nerve cells, it computes the matching score, that is, the output values of the output nerve cells, through the feed forward connecting weights and sample input. The nerve cell activity with the largest matching score is enhanced through the lateral inhibiting between the output nerve cells. The other output nerve cells activity degrees are gradually decreased. Nerve cells represent random variables, the edges among nerve cells represent the relationship between cells. The conditional probability express relationships strength. The variable can be the abstract of any problem, such as test value, observations, etc. The feedback connecting from the output nerve cell to the input nerve cell performs learning and comparing. ART neural network model can be described with the differential equation and perform self-organization with the competitive learning[13].The adjacent processing units restrain one another, finally, only one processing unit is active, this active processing unit represents the present type of the fault in power system. Therefore, it is applicable to express and analysis of uncertainty and probabilistic events, applied to a conditional relies on multiple controlled factor of decision making, can make reason among incomplete, inaccurate or uncertain knowledge or information.

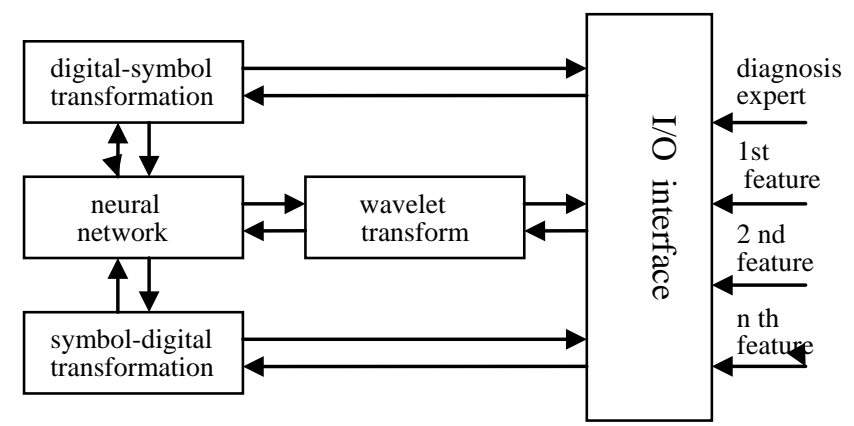

Fig.2: The schematic diagram of the power system fault diagnosis

The architecture of the ART neural network model can be divided into the caring subsystem and adjusting subsystem[14]. By means of the two mutual compensating subsystems and interaction 
of the controlling mechanism, the fault data is obtained. The caring subsystem solves the evaluation of the power system performance in the known environment, for example, the line, transformer and bus. The adjusting subsystem solves the uncertainty and incomplete in the power system. The following kind of system is considered. Because the ART neural network model is the kind of selforganizing neural network model, it forms the response to input through competition. In the application of the neural network to the fault diagnosis of the power systems, we need the preprocessing network that the features characterizing original signals are extracted.

Learning the neural network, we can obtain the qualitative information and quantitative information. The qualitative information is reflected by arc, representing the relationships among variables; the quantitative information is reflected by relationship strength among variables, namely represented by the table of conditional probability. Uncertainty reasoning methods mostly developed on the basis of uncertainty reasoning method, or rather to expand or improve certainty reasoning. The most common way is to put instructions degree of certainty data attached to reasoning rules. Neural network reasoning actually know results obtained parameters process, fault diagnosis reasoning is to obtain the reason of fault by using the fault information, says variables and the state of the fault occurred in event joint probability is the largest. The fault diagnosis model established with neural network can clearly express the relationship among components, all levels of the protection and the breakers.

\section{Simulation and conclusion}

In order to demonstrate the fault diagnosis method established with neural network in the power system, the following conditional parameters are selected. The electric power system fault diagnosis includes: line, transformer and bus. Relay protection of lines and transformer protection is 3sectional protection: main protection $(\mathrm{m})$ only protect circuit itself, the scope of protection is generally 75 of the full-length. The first backup protection (p) protecting the whole length of circuits, when the main protection didn't start, the first backup protection start automatically. The second backup protection (s), the adjacent elements fault but the protection doesn't start, the second backup protection start automatically to remove fault. Bus relay protection is only main protection, when the bus is fault, relay protection start automatically.

In this paper, three types of faults are analyzed. We do 3-scale wavelet transform on 256 data for every fault sample and extract feature vectors as discussed above. At first, we regard $A_{-1}^{d} f$ of every sample as a learning set and input it to neural network, then we regard $A_{-2}^{d} f$ and the mean value, mean square value and variance of $A_{-2}^{d} f$ every sample as a learning set and input it to neural network. Among the faults diagnosis, the maximum fault probability is obtained, therefore, the diagnosing result is obtained and given in Table 1 . From the result, we can see that by both of the two feature extraction methods, the three types of faults are identified correctly.

The features extracted from wavelet transform can characterize original signals accurately with the data quantity greatly compressed. At the same time, the noise is restrained. If these feature vectors are used as neural network input sets, the construction of the neural network will be terrible simplified and the calculation time obviously shortened. This makes it possible to use this method to on-line fault diagnosis. It is proved by experiments that this measure is especially efficient in diagnosing some faults.

Table 1: Diagnosing Result

\begin{tabular}{|c|c|c|c|c|}
\hline No. & fault1 & fault2 & fault3 & diagnosis \\
\hline 1 & 0.91203 & 0.00870 & 0.03768 & fault1 \\
\hline 2 & 0.00836 & 0.97651 & 0.01829 & fault2 \\
\hline 3 & 0.00521 & 0.02512 & 0.93707 & fault3 \\
\hline
\end{tabular}

\section{References}


[1] Jiang Qingxian. Robust Estimation for FOG Data Based on Wavelet Threshould De-Noising. Chinese Journal of Sensors and Actuators, vol.22, pp. 1595-1601, November, 2009.

[2] Song Ningfang. Wavelet Variance Analysis of Random Error Properties for Fiber Optic Gyroscope. Infrared and Laser Engineering, vol.39, pp. 924-928, May, 2010.

[3] Liu Lisheng. Post-Flight Data Processing of Trajectory Measurement. Beijing:National Defence Industry Press, pp. 298-300, 2000.

[4] Guo Lihua. Application of Wavelet transform in Random Error Separation of Telemetered Data. Journal of Projectiles, Rockets, Missiles and Guidance, vol. 31, pp. 249-252, August, 2011.

[5] Xia Qing. Analysis of Random Errors of Radar Measurement data Based on Wavelet transformation. Journal of Spacecraft TT\&C Technology, vol. 25, pp. 77-80, October, 2006.

[6] Zhou Lifeng. A Study on Error Analysis of Radar Measurement Data in Proving ranges. Radar \& Ecm, pp.4-7, 2008.

[7] Wang Xinlong. Investigation of Random Error Model for Fiber Optic Gyroscope. Journal of Beijing University of Aeronautics, vol.32, pp. 769-772, July, 2006.

[8] Guofeng Yang. Research on Power System Fault Diagnosis Based on Bayesian Network. Proceedings of the ICCSEE2013, pp. 2553-2556, March, 2013.

[9] Wen Fushun. Fault Sections Estimation in Power System Using Genetic Algorithm and Simulated Annealing. Proceedings of the CSEE, pp. 29-35, May, 1994.

[10] Xiuyun Duan. Research on Method of Random Error Separation Based on Wavelet Transform and Frequency bands Character. Proceedings of the ICCSEE2013, pp. 2525-2528, March, 2013.

[11] Wu Yousheng. The Application of Wavelet Transform and Artificial Neural Networks in Machinery Fault Diagnosis. Proceedings of the ICSP’96, pp. 1609-1612, Oct., 1996.

[12] Dong Chen. Applications and Research of the Bayesian Network in Power System Fault Diagnosis. Shenyang University of Technology Press. 2009.

[13] Feng Ding. Neural Network Expert Systems. Beijing: Science Press, 2006.

[14] Shie-Jue Lee. An ART-Based Construction of RBF Neural Networks. IEEE Transactions on Neural Networks, vol.13,no.6,pp.1308-1321, Nov.,2002. 